\title{
Spinal muscular atrophy type I and the dual role of viruses: An interview with Professor Basil T. Darras, Professor of Neurology (Pediatrics) at Harvard Medical School
}

\author{
IOANNIS N. MAMMAS and DEMETRIOS A. SPANDIDOS \\ Department of Clinical Virology, School of Medicine, University of Crete, 71003 Heraklion, Crete, Greece
}

Received December 20, 2017; Accepted February 7, 2018

DOI: $10.3892 /$ etm.2018.5884

\begin{abstract}
According to Professor Basil T. Darras, Professor of Neurology (Pediatrics) at Harvard Medical School and Director of the Spinal Muscular Atrophy (SMA) Program at Boston Children's Hospital in Boston (MA, USA), the diagnosis of SMA type I is clinical and is based on detailed general physical and neurological examinations. SMA type I remains the most common genetic disease resulting in death in infancy and is really devastating for the child, the parents, as well as the medical professionals with the privilege of caring for patients with SMA and their parents. The proposed management options include: i) no respiratory support; ii) non-invasive ventilation; and iii) tracheotomy with mechanical ventilation. Deciding, which option is the best, is indeed a very personal decision. The optimal clinical care should be extremely mindful of parents' wishes and management goals with regard to the quality of life. Since the end of 2016 in the USA, and recently in Europe, there exists the possibility of accessing a novel treatment drug for SMA, namely Nusinersen. This antisense oligonucleotide is administered intrathecally and increases the production of the fully functional SMN protein, thus improving motor function, the quality of life and survival. Among the ongoing clinical trials, oral treatment with RG7916, a small molecule $S M N 2$ splicing modifier, appears to be really promising. Gene therapy using viral vectors is expected to offer an 'one and done' therapy and possibly a cure, if administered early in life, before any symptoms appear. It is really interesting that viruses, which at the moment are the cause of death of children with SMA, if genetically modified, may be used for their treatment.
\end{abstract}

Correspondence to: Professor Demetrios A. Spandidos, Department of Clinical Virology, School of Medicine, University of Crete, 71003 Heraklion, Crete, Greece

E-mail: spandidos@spandidos.gr

Key words: spinal muscular atrophy, SMA type I, Professor Basil T. Darras, gene therapy, viruses

\section{Contents}

1. Introduction

2. Questions and Answers

\section{Introduction}

A 3-month old breast-feeding child with a weight of $4 \mathrm{~kg}$ presented at the local Pediatric Accident and Emergency Department accompanied with his parents due to a prolonged coughing episode. The physical examination of the child revealed no weight gain after the 30th day of his life, profound weakness and hypotonia of his upper and lower limps and a weak cry. The diagnosis of spinal muscular atrophy (SMA) type I was included in the differential diagnosis and the child was admitted to the Pediatric Ward, where he was evaluated by the Specialist Consultant in Pediatric Neurology and specific blood tests were sent for analysis by polymerase chain reaction (PCR). The child was discharged two days later, with nasogastric (NG) tube-feeding. No formal information was provided to his parents, who were waiting for the genetic diagnosis, until only two weeks later when the results of genetic testing came back positive and the diagnosis of SMA type I was established.

SMA type I remains the most common genetic disease resulting in death in infancy (1-6). Characterized by progressive motor and respiratory muscle weakness, this autosomal recessive neuromuscular disorder is caused by low levels of the survival motor neuron protein (SMN) due to inactivating bi-allelic deletions and other disabling mutations in the survival motor neuron $1(S M N 1)$ gene $(5,6)$. Respiratory failure, due to increased pulmonary muscle weakness and secretions induced by common viral infections, such as respiratory syncytial virus (RSV) infection, is the leading cause of morbidity and mortality in children with SMA type I (7). The diagnosis should be confirmed by an expert in pediatric neuromuscular diseases; invasive investigations are not usually necessary as the diagnosis is confirmed with a DNA-based blood test for SMN1 deletions and other mutations (3). Due to its clinical similarity with congenital myopathy, clinicians have indeed difficulties in its rapid and direct diagnosis (5). The timing of the final diagnosis in cases in which SMA type I is suspected is crucial, as early diagnosis may lead to early supportive care and reduction in parental stress (8). Pre-conception and pre-natal genetic screening for SMA type I 
should be performed in families with children affected by SMA type I (9); to date, several studies have evaluated the necessity and feasiblity of expanded newborn screening in the general population $(10,11)$. In the future, this perspective can be enhanced by the minimization of the cost of SMA detection through newborn screening, which will allow SMA to be added to the current newborn screening programs (12).

SMA type I is usually fatal in the first two years of life $(1,2,13)$. The supportive care of children should be delivered close to home by a multidisciplinary team aiming to optimize the children's quality of life and to provide access during times of crisis $(2,3)$. The long-term survival outcome is determined by the choice of different management options, such as tracheostomy and invasive mechanical ventilation (TV) or continuous non-invasive respiratory muscle aid (NRA), including non-invasive ventilation with bi-level positive airway pressure (Bi-PAP), which both can prolong survival (13). However, different scientific opinions exist for the role of these options in the optimal management of children with SMA $(3,14,15)$. At the moment, the newly approved treatment drug, Nusinersen, may alter the morbidity and mortality and may hence improve the quality and duration of the child's life $(11,16)$. Additionally, with further investigational drugs for SMA type I now in clinical trials and with improvements in novel therapeutic approaches, such as gene therapy using viral vectors $(11,17)$, the future is definitely expected to be optimistic. However, there continue to be complex treatment and ethical challenges, which need to be considered regarding the enrollment of children with SMA type I into current clinical trials $(18,19)$. Moreover, to date, not all patients with SMA type I are accessed by specialist healthcare services and these patients may not have access to research opportunities and optimal care (20). Interestingly, gene therarpy using viral vectors is included in the list of the future therapeutic solutions, yielding promising results in early clinical trial phases (16). Future research in this field will answer the question of whether viruses will continue to be included in the life-threatening causes of morbidity and mortality in children with SMA type I or will efficiently be used for the gene therapy of SMA and other genetic diseases.

Currently, one of the leading top scientists worldwide on SMA is Professor Basil T. Darras (Fig. 1), Professor of Neurology (Pediatrics) at Harvard Medical School and Director of the SMA Program at Boston Children's Hospital in Boston, MA, USA. As it is highlighted in the official site of Boston Children's Hospital (http://www.childrenshospital.org), during his childhood in Greece, his parents instilled in him intellectual curiosity, the ambition to excel, and personal values focused on service. His approach to care reflects his interest in the genetic basis of neurological disease and his desire to practice on the forefront of clinical care and research. Born and raised in Greece, he earned his country's highest marks on nationwide tests to be admitted to medical school and, in 1977, he graduated from the School of Medicine, University of Athens, at the top of his class. He then moved to the USA and trained in Pediatrics and Pediatric Neurology at the Nassau County Medical Center (Stony Brook, NY, USA) and in Tufts Medical School - New England Medical Center (Boston, MA, USA), respectively. He also completed a post-doctoral fellowship in Genetics at the Yale University School of

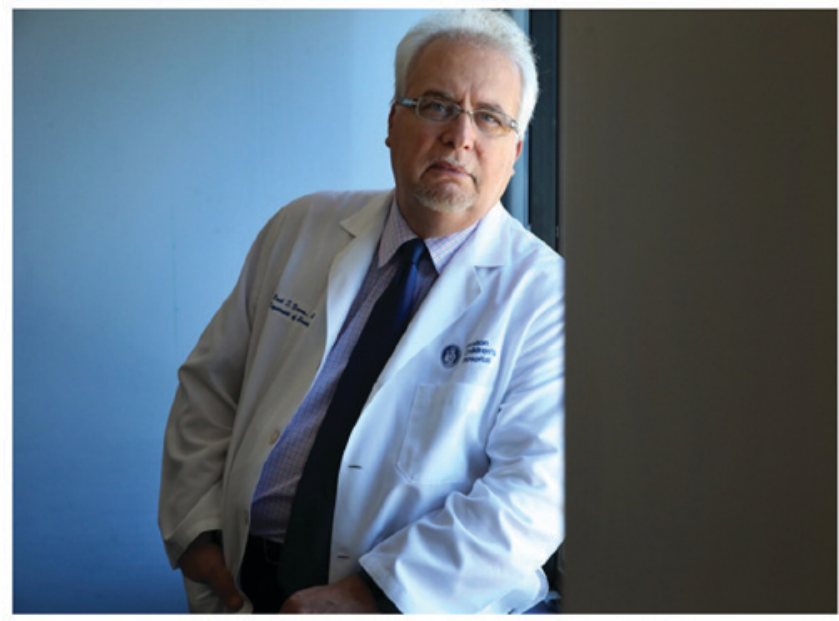

Figure 1. Professor Basil T. Darras, Professor of Neurology (Pediatrics), Harvard Medical School, and Director of the SMA Program at Boston Children's Hospital in Boston, MA, USA.

Medicine (New Haven, CT, USA). To date, he is associate neurologist-in-Chief at Boston Children's Hospital and he holds the Joseph J. Volpe Chair in Neurology at Harvard Medical School. He is the Chief of the Division of Clinical Neurology in the Department of Neurology at the hospital. For 11 years, he ran the hospital's neurology residency training program and he was twice voted teacher of the year by neurology residents. His special focus is on the care of children with neuromuscular conditions originating from inherited or acquired conditions of the motor unit. These include patients with complex muscle diseases, such as muscular dystrophies and congenital or metabolic myopathies, neuromuscular transmission defects, neuropathies, motor neuronopathies, as well as inflammatory muscle or nerve conditions. Furthermore, he sees patients with general neurological problems, such as seizures, when he attends on the inpatient neurology services.

His research efforts encompass both clinical and basic projects focused on molecular genetics, diagnostics and therapeutics for neuromuscular diseases in children. At Boston Children's Hospital, he directs two innovative multi-disciplinary clinical programs: The Neuromuscular Center, which provides diagnostic evaluation and therapeutic services for children with neuromuscular diseases and is the largest of its kind in the country, and the SMA Clinical and Research Program, which seeks to improve medical care of children with SMA and discover new treatments for this devastating motor neuron disease. He has edited the only major, comprehensive textbook on pediatric neuromuscular disorders, entitled 'Neuromuscular Disorders of Infancy, Childhood, and Adolescence: A Clinician's Approach', the most comprehensive text in pediatric neuromuscular diseases ever published, with more than a thousand copies sold worldwide in the first few months after the release of the second edition in 2015. He is also the author of over 190 peer-reviewed publications and over 80 book chapters.

Professor Darras is indeed a great clinician and top scientist, who through his clinical and research experience, as well as his hospital leadership roles, has influenced the care in the field of pediatric neuromuscular diseases both in the USA, as well as internationally and has obtained a worldwide reputation. At the same time, Professor Darras is a great humanitarian, and this 
was proven by his interest in our young patient from a Greek island; from the first hour after the diagnosis, he offered state-of the-art advice and support. In the context of the 17th Annual Pediatric Neurology Congress, which was organized by the Hellenic Pediatric Neurology Society in Athens, Greece, on December 8, 2017, Professor Darras gave his plenary lecture on 'Targeting the faulty genes in neuromuscular disorders'.

\section{Questions and Answers}

Question: What is SMA, where is the deficit and what is the current prognosis of our little patient from a Greek island, who was just diagnosed with SMA type I?

Answer: SMA is an autosomal recessive genetic disorder, which is characterized by the progressive degeneration and loss of motor neurons in the spinal cord and brain stem nuclei. It is caused by homozygous deletions involving exon 7 and other disabling mutations in the SMN1 gene at locus 5q13.2. These mutations result in a reduced amount of functional full-length SMN protein, which is a major subunit of the spliceosomal complex, highly important in RNA processing. A nearly identical gene, SMN2, also located on chromosome 5q13.2, harbors an exonic splicing enhancer mutation that limits the inclusion of exon 7 during splicing events. The SMN2 gene copy number and the level of SMN protein are important modifiers of disease severity. SMA in children is a clinical continuum, divided into three broad clinical types based on maximal motor function achieved: SMA type I, SMA type II and SMA type III. The onset of SMA type I is before the age of 6 months and these children are 'non-sitters'; they never achieve the ability to sit independently. SMA type II, with onset between 6 and 18 months, defines the category for 'sitters' and SMA type III for 'walkers'. A wide spectrum of severity exists within each of these types. A shortened lifespan is typical for children with SMA type I; in the past, most type I patients did not survive beyond the age of 2 years. However, with improved respiratory and nutritional supportive care, survival has improved in recent years.

Question: How sure can you be for the diagnosis of SMA type I clinically? Should parents be informed immediately about the diagnosis of their child based on the clinical examination by an expert in pediatric neuromuscular diseases before the final PCR result?

Answer: The diagnosis of SMA type I is clinical and is based on detailed physical and neurological examinations. SMA type I is characterized by severe, proximal and symmetric muscle weakness, lack of motor development, poor muscle tone and hyporeflexia or areflexia. Weakness of the intercostal respiratory muscles with the relative preservation of the diaphragm musculature leads to characteristic bell-shaped chest and paradoxical respiration, known as abdominal breathing. The muscles of the face are relatively spared; the diaphragm is not involved until late in the course of disease. Fasciculations of the tongue are seen in most, but not all children. Among these clinical signs, areflexia, paradoxical respiration and tongue fasciculations are indeed very strongly suggestive of SMA type I prior to a positive PCR result. Parents should be informed immediately as soon as the results of genetic testing become available. There are atypical cases, however, in which the diag- nosis is not certain and more investigations are required, such as electromyography (EMG) and nerve conduction studies prior to genetic testing. Non-5q13-associated SMAs comprise a heterogeneous group of motor neuron diseases associated with mutations in a variety of different genes. Patients with these disorders generally exhibit clinical characteristics that help us to differentiate them from the 5q13-associated SMA.

Question: The diagnosis of SMA type I is definitely a devastating experience for the child, the parents and the whole family, suddenly impacting their whole lives. How difficult is to inform the parents about the diagnosis of SMA type I?

Answer: Yes, this is indeed a devastating diagnosis. And it is really devastating for the smiling infant, who progressively develops weakness, hypotonia and respiratory distress, related to respiratory failure and life-threatening infections caused by common pathogens, such as viral respiratory infections. It is devastating for the unsuspecting parents, who listening to the weak cry of their child, have to deal with so many dilemmas, which are difficult to be answered even by experienced pediatric consultants. It is, indeed, very challenging for the pediatric consultant, who suspects for the first time this disease, for the pediatric neurologist, who has to report the diagnosis to the parents after the positive genetic testing result becomes available, for the medical professionals with the privilege of caring for these patients and their parents, as well as for the primary care physician, as in your patient, who on a Greek island has to replace the multidisciplinary team required for the health care of this child at home after the diagnosis.

Question: According to several studies in the literature, diagnostic delay is common in SMA type I. Based on your experience, how prepared are indeed general primary care pediatricians for this diagnosis? What is the impact of this delay to child's outcome? Answer: For prime care pediatricians this is an extremely rare diagnosis. Only one child with this diagnosis is usually seen during the whole career of a general primary care pediatrician. Medical training is essential. Our medical students and residents at Boston Children's Hospital and Harvard Medical School may have the opportunity to examine children with SMA of different types; this experience is indeed very educational and undoubtly unforgettable for their whole professional life. The early diagnosis and employment of management interventions for these children are indeed essential and will become even more important in the future as more treatments for SMA become available.

Question: SMA type I is the most common genetic cause of infant mortality. I would like to have your comment on the necessity of genetic counselling in the parents of our young patient.

Answer: There is a $25 \%$ risk that each offspring of two carrier parents will be affected with the autosomal recessive inherited forms of SMA. Genetic testing can be done prenatally by amniocentesis and chorionic villus sampling to look for SMN1 gene deletions. This is called prenatal genetic diagnosis. Parents can then decide to abort an affected fetus. In vitro fertilization (IVF) and pre-implantation genetic diagnosis is an alternative option, which can be carried out in this family. Embryos are tested to see if they are affected by SMA and unaffected embryos can be transferred to the uterus. Chorionic 
villus sampling may then be used later in the pregnancy to confirm that the growing fetus is unaffected. Research has shown that non-invasive analysis through testing of circulating fetal cells in the mother's blood may also be possible in the future. Genetic counselling is really a very positive issue that should be immediately explained and proposed to these parents.

Question: Parents had requested newborn screening for a list of rare diseases, including metabolic and genetic diseases. In this list, SMA was absent. When is newborn screening for SMA going to be included?

Answer: The newborn screening test in the general population is a very useful tool, which in the future needs to be approved. This is of great interest as this will help identify pre-symptomatic individuals in the general population enabling us to initiate early therapy for children with SMA. At the moment cost-effectiveness issues regarding newborn screening for SMA are under discussion, but it has been approved in a number of states in the United States including Massachusetts.

Question: So, now, what are the proposed management options in our young patient from Greece, who was just diagnosed with SMA type I?

Answer: There are three management options. The first one includes no respiratory support; the second includes non-invasive ventilation with Bi-PAP, and the third option tracheotomy with mechanical ventilation. These options should be discussed with the parents or care providers before respiratory failure occurs. The type of respiratory support is dependent on the individual's respiratory status, 'quality of life' goals and reduction in respiratory complications. Palliative care, with or without non-invasive ventilator support, is an option for the most severely affected individuals. Indications for non-invasive ventilation in SMA include hypoventilation, as demonstrated by decreased oxygen saturation by pulse oximetry, or obstructive sleep apnea. Non-invasive ventilation with Bi-PAP has been shown to improve chest wall and lung development and is proposed to reduce lung infections and pulmonary comorbidity in SMA. Tracheotomy for chronic ventilation is a controversial option that can be discussed with parents. Airway clearance can also be addressed with a mechanical in-exsufflator in conjunction with suctioning and chest physiotherapy, especially in the setting of acute illness, reducing pulmonary complications.

Question: Which one do you believe is the best option for our little patient?

Answer: This is a personal decision, depending on parental preference. Deciding which proposed option is the best option that parents want to request is indeed a very personal decision. As I mentioned earlier, parents should be informed for all these three options in detail and they should be asked to decide. In the USA, parents usually choose the second option, but this depends on how aggressive they want to be in treating the child. This also depends on the available medical services that can be offered to them. In our little patient, I would suggest home-based health care under your coverage and support, with open access to the University Pediatric Ward in Athens, during times of acute febrile illnesses. Definitely, this child should not end up with a tracheostomy and long-term mechanical respiratory support in one of your Pediatric Intensive Care
Units (PICUs). Personally, I don't support this option in children with SMA type I, but, again, this is a personal decision that parents have to make.

Question: In our patient, NG tube-feeding led to $50 \mathrm{~g}$ weight gain per day and this led to an impressive neurological improvement.

Answer: Nutritional care is a significant issue in this child. Bulbar dysfunction is universal and can lead to failure to thrive and aspiration. The use of flexible NG tubes can be helpful. Gastrostomy can also improve the child's nutritional status and decrease long-term aspiration events; however, this should be considered early in the course of the disease. Consultation with a specialized dietician, who is aware of the special needs and concerns of SMA patients, is also very useful.

Question: According to your recent review article on SMA published in Pediatric Neurology (21), the long-term care of these children should be delivered by a multidisciplinary team. What happens when there are no such multidisciplinary teams, e.g., in our Greek island, or in countries under conflict? What is currently the standard of care for children with SMA in Boston? Answer: A recent worldwide literature search has demonstrated that, even in 2017, not all children with SMA type I have access to specialty healthcare services; these patients may not have access to research opportunities and optimal care as well. In Boston, I have the opportunity to direct our SMA Program at Boston Children's Hospital, which is devoted to providing comprehensive care for children with SMA. This program brings together specialists from a variety of disciplines including Neurology, Genetics, Orthopedics, Physical Therapy, Respiratory/Pulmonary, Nutrition, Gastroenterology, Anesthesiology, Surgery and Social Work. We hold a dedicated SMA clinic once a month, which allows parents to meet with all of the child's SMA-related care providers during a single visit. These specialists are experienced in caring for children with SMA. For example, children with SMA may develop complications when having anesthesia because of respiratory muscle weakness. Our team of anesthesiologists understands SMA and has the expertise to safely provide anesthesia to our SMA patients. Moreover, we evaluate a child's health as a whole and work with the family as a team to make the best treatment decisions. For families whose children are facing an especially difficult prognosis, we put them in touch with the Pediatric Advanced Care Team at Boston Children's. This group of professionals can provide comfort and support to the parents as they make decisions about the extent of the treatment. Our clinic also gives parents the chance to meet other families affected with SMA and to learn about research opportunities.

Question: SMA type I and quality of life. Should the care of children with SMA, after the diagnosis is made, be hospital- or home-based?

Answer: The quality of life of these children should remain our main target. So, definitely, their long-term multidisciplinary health care should be home-based. However, as I mentioned earlier, there should be a specific protocol of hospital admission in place, in case of an acute illness that will require admission of the child to the hospital. Optimal clinical care for 
these patients and our management goals should be extremely mindful of the issues regarding the quality of their lives.

Question: To date, several studies have demonstrated that the long-term survival outcome is determined by the choice of the treatment. How possible is the creation of a standard medical decision tool? Are parents really ready for such short-term difficult decisions?

Answer: To date, there are several consensus efforts, as well as advocacy groups, such Cure SMA, that are extremely helpful for both the medical teams, who are responsible for providing the standard of care and support for these children, as well as for their parents.

Question: You mentioned before that, in general, you don't support the option of long-term invasive ventilation in children with SMA type I. How frequent was this practice in the past? Answer: Yes, although I have been offering this option to the families for many years, thus far only a minority of families have opted for long-term invasive ventilation, while waiting for the discovery of a treatment of this disease. However, this is really not realistic given the presumed dramatic loss of anterior horn cells and probably other neural structures in these ventilator-dependent children over time. For this reason, these children may not be good candidates for any future treatments, although that is not totally certain. We have to be realist and this information is more than essential, when talking to the parents about various options.

Question: Non-invasive ventilation with Bi-PAP can prolong survival; however, its prolonged usage is also a subject of a debate. Can this option be offered at home in Boston?

Answer: Respiratory failure is the major cause of mortality in children with SMA type I. For this reason, for children whose parents have chosen the second management option, non-invasive ventilation, when combined with nutritional support, can indeed prolong survival. Our hospital offers to these patients the Critical Care, Anesthesia Perioperative Extension (CAPE) and Home Ventilation Program. This program, which started in 2007, provides comprehensive services to help families plan for their child's discharge from the hospital and manage respiratory care at home. The program also evaluates home respiratory equipment and is available $24 \mathrm{~h}$ a day for consultation and support. These services are intended to optimize care at home, facilitate any hospital-based care and thus prevent more admissions to the hospital.

Question: Respiratory infections can be life-threatening for children with SMA type I. Which viral infections are more dangerous for the life of these children? What are the recommended prevention strategies against viral infections for children with SMA type I?

Answer: Yes, any child with SMA type I is at risk to develop pneumonia which, once established, is very difficult to treat. For this reason, when a child presents with fever, should be covered with broad-spectrum antibiotics. Common viral respiratory infections, which can be life-threatening, are RSV and seasonal influenza viruses. Prevention of these viral infections is strongly recommended, including seasonal influenza vaccination of the child and its sibling, isolation from the ill sibling, as well as RSV prophylaxis. Vaccination scheme should not be discontinued after the diagnosis of SMA, if anything, it should be enhanced.

Question: Following regulatory approval of the first drug treatment for SMA in the USA and recently in Europe and other countries, the prospects for care for patients with SMA are expected to change. What are the prospects of this recently introduced into clinical practice treatment for SMA?

Answer: Since the end of 2016 in the USA and since two months ago in Europe, there is now the possibility of having access to the new treatment for SMA called Nusinersen, also known by its brand name Spinraza ${ }^{\mathrm{TM}}$. This treatment is offered to children with SMA type I, when the child is eligible for the treatment and both the child's medical team and the child's parents or guardians agree that it could be of potential benefit. This innovative treatment is an antisense oligonucleotide, which compensates for the effects of the SMN1 mutations by binding to and hence inactivating an intronic splicing silencer in the 'backup' gene $S M N 2$. This results in an increase of exon 7 inclusion during splicing of the SMN2 mRNA and, therefore, increases the production of fully functional SMN protein. Nusinersen has been shown that can improve motor function and acquisition of developmental milestones in younger patients, as well as the quality of life and survival of these children.

Question: What is your experience in Boston with Nusinersen? Answer: Boston Children's Hospital in Boston has been involved in the clinical trials of Nusinersen, sponsored by Ionis Pharmaceuticals, since they were first initiated in 2011. We were the first in the world to enroll a child with SMA type I in the phase 3 ENDEAR trial, in 2014. This trial enrolled infants under 7 months of age and randomly assigned them to receive Nusinersen by spinal tap (two thirds) or a sham procedure (one third). The trial was interrupted in August 2016 for an interim analysis. The results showed that $40 \%$ of infants given Nusinersen, versus none of the control ones, achieved significant improvement in the acquisition of motor milestones, such as head control, rolling over, sitting, standing and, in a few cases, walking with support. A second trial, called CHERISH, evaluated Nusinersen in children with type II SMA ages 2 to 12 years, who had the ability to sit independently, but not walk independently. Children receiving Nusinersen showed improved motor function, and this trial was interrupted as well, in November 2016. Because of these positive results, the FDA gave Nusinersen priority review status and approved the drug in December 2016 for all forms of SMA.

Question: How is it administered and for how long?

Answer: Nusinersen should be administered intrathecally. It is injected directly into the spinal canal requiring a lumbar puncture also known as spinal tap. The initial treatment includes 4 loading doses, and then a maintenance dose, once every 4 months. This treatment will most likely need to be given for life.

Question: Can Nusinersen give hope to our little patient from Greece?

Answer: I think yes, if this child is treated with Nusinersen will probably benefit from it. Currently, this treatment can be offered at the 'Aghia Sophia' Children's Hospital by Dr Roser Pons, 
Assistant Professor of Pediatric Neurology at the University of Athens. Following this newly approved treatment, our 3-month old child's developmental milestones and motor function will probably improve. This is, however, an extremely expensive medication and it is a treatment [therapia $(\theta \varepsilon \varrho \alpha \pi \varepsilon i ́ \alpha)$ in Greek],

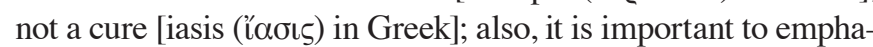
size that not every child, who gets treated with Nusinersen, improves. It seems that early treatment leads to better outcomes.

Question: At the moment, there are several experimental therapeutic options that are under investigation in your Center, as well as worldwide. Among these clinical trials that are currently ongoing, could anyone be an alternative option and give hope to our little patient from Greece?

Answer: Yes. There are currently several clinical trials, including gene therapy using viral vectors. Indeed, the pediatric community remains alert to the rapidly changing developments in the treatment of SMA; as current research suggests, improved outcomes may soon be forthcoming. I don't think that when gene therapy trials get initiated, the age of the patient from your island will be acceptable in order to be included. Among the existing clinical trials, if parents want to try an experimental therapy, I would advise them to try oral therapy with RG7916, which is really quite promising. This is a small molecule modifier of SMN2 splicing, leading to an increase in SMN2 full length transcript and thus functional SMN protein. Preliminary clinical data from Roche and the SMA Foundation have shown that RG7916 has a favorable preliminary safety profile and it is generally well tolerated. As I know, this clinical trial is currently ongoing in Rome, Italy, a country close to Greece; this small molecule will be administered to the child orally and will be free of charge for the parents. RG7916 is not an FDA or EMA approved drug yet and its safety profile is still under investigation. For this purpose, you should contact Professor Eugenio Mercuri, Head of the Pediatric Neurology Unit of the Catholic University in Rome.

Question: In the literature, there are several reported ethical issues that need to be considered at the enrollment of children with SMA type I into clinical trials. How do you manage these issues?

Answer: With several investigational drugs for SMA type I, now in clinical trials, there are indeed several ethical issues. These issues arise at the enrollment of patients, especially in cases when placebo treatment is going to be administered, as well as during the interpretation of the analysis results of clinical trials. For this reason, in the USA, as well as in other countries like the European ones, targeted workshops take place and specialized committees review these issues frequently; the criteria that are established are very strict and are closely evaluated and monitored. The collaboration between clinicians and researchers working together from different centers has led to the initiation of multicenter trials, for which the best outcome measures and methodologies are utilized. Hippocrates' saying in his book entitled 'Epidemics I', almost 2,500 years ago, 'to do good or to do no harm', is indeed a fundamental principle of great value, which is always relevant and up-to-date.

Question: And our last question. SMA type I and gene therapy using viral vectors. How optimistic are you about this option?
Can viruses, which at the moment are included in the list of the most common causes of morbidity and mortality in children with SMA type I, be efficiently used for the SMA gene therapy?

Answer: Yes, advanced low-risk viral vectors are expected to lead to breakthroughs in the field of gene therapy. Self-complementary adeno-associated virus type 9 (scAAV-9) is the primary viral vector under investigation to deliver the SMN1 transgene. Published results have shown marked improvement in a small cohort of children treated with gene therapy. The reason that FDA hasn't approved gene therapy for SMA type I yet was probably the limited number of children enrolled in the initial clinical trial. The production of the required viral vectors was performed in a local Virology Research Lab, but now the obstacle of large-scale production has been overcome with adequate viral vector production by a recently created industrial-type facility. The future is full of hope and I am very optimistic that we will have a number of treatments for SMA. The cure of SMA does not seem as far into the future as it looked 5 or 10 years ago. Gene therapy using viral vectors is expected to offer an 'one and done' therapy and possibly cure the disease if administered early in life before symptoms appear. And it is really interesting, as you noted, that viruses, which at the moment can be the cause of death in these children, with proper genetic engineering, could be used for their treatment in the future. This can be a very interesting subject in one of your future Workshops on Pediatric Virology.

Question: Thank you for your answers, your interest and your help with our little patient from Greece.

\section{References}

1. Darras BT: Spinal muscular atrophies. Pediatr Clin North Am 62: 743-766, 2015.

2. Darras BT, Monani UR and De Vivo DC: Genetic disorders affecting the motor neuron: Spinal muscular atrophy.In: Swaiman's Pediatric Neurology: Principles and Practice. Swaiman KF, Ashwal S, Ferriero DM, Schor NF, Finkel RS, Gropman AL, Pearl PL and Shevell MI (eds). Elsevier, pp1057-1064, 2017.

3. Roper H and Quinlivan R; Workshop Participants: Implementation of "the consensus statement for the standard of care in spinal muscular atrophy' when applied to infants with severe type 1 SMA in the UK. Arch Dis Child 95: 845-849, 2010.

4. Ogino S and Wilson RB: Spinal muscular atrophy: Molecular genetics and diagnostics. Expert Rev Mol Diagn 4: 15-29, 2004.

5. Faravelli I, Nizzardo M, Comi GP and Corti S: Spinal muscular atrophy - recent therapeutic advances for an old challenge. Nat Rev Neurol 11: 351-359, 2015.

6. Bowerman M, Becker CG, Yáñez-Muñoz RJ, Ning K, Wood MJA, Gillingwater TH and Talbot K; UK SMA Research Consortium: Therapeutic strategies for spinal muscular atrophy: SMN and beyond. Dis Model Mech 10: 943-954, 2017.

7. LoMauro A, Aliverti A, Mastella C, Arnoldi MT, Banfi P and Baranello G: Spontaneous breathing pattern as respiratory functional outcome in children with spinal muscular atrophy (SMA). PLoS One 11: e0165818, 2016.

8. Lin CW, Kalb SJ and Yeh WS: Delay in diagnosis of spinal muscular atrophy: A systematic literature review. Pediatr Neurol 53: 293-300, 2015.

9. Kekou K, Sofocleous C, Konstantinidis G, Fryssira H, Mavrou A, Kitsiou S and Kanavakis E: SMA prenatal diagnosis: A modified protocol to help differentiation between deletions and gene conversion. Mol Cell Probes 29: 71-73, 2015.

10. Verhaart IEC, Robertson A, Leary R, McMacken G, König K, Kirschner J, Jones CC, Cook SF and Lochmüller H: A multi-source approach to determine SMA incidence and research ready population. J Neurol 264: 1465-1473, 2017. 
11. Boardman FK, Young PJ and Griffiths FE: Newborn screening for spinal muscular atrophy: The views of affected families and adults. Am J Med Genet A 173: 1546-1561, 2017.

12. Phan HC, Taylor JL, Hannon H and Howell R: Newborn screening for spinal muscular atrophy: Anticipating an imminent need. Semin Perinatol 39: 217-229, 2015.

13. Gregoretti C, Ottonello G, Chiarini Testa MB, Mastella C, Ravà L, Bignamini E, Veljkovic A and Cutrera R: Survival of patients with spinal muscular atrophy type 1. Pediatrics 131: e1509-e1514, 2013.

14. Panitch HB: Counterpoint: Is noninvasive ventilation always the most appropriate manner of long-term ventilation for infants with spinal muscular atrophy type 1? No. Chest 151: 965-968, 2017.

15. Bach JR Jr: Point: Is noninvasive ventilation always the most appropriate manner of long-term ventilation for infants with spinal muscular atrophy type 1? Yes, almost always. Chest 151: 962-965, 2017.

16. Scoto M, Finkel RS, Mercuri E and Muntoni F: Therapeutic approaches for spinal muscular atrophy (SMA). Gene Ther 24: 514-519, 2017.

17. Singh P, Liew WK and Darras BT: Current advances in drug development in spinal muscular atrophy. Curr Opin Pediatr 25: 682-688, 2013.
18. Tizzano EF and Finkel RS: Spinal muscular atrophy: A changing phenotype beyond the clinical trials. Neuromuscul Disord 27: 883-889, 2017.

19. Finkel RS, Bishop KM and Nelson RM: Spinal muscular atrophy type I: Is it ethical to standardize supportive care intervention in clinical trials? J Child Neurol 32: 155-160, 2017.

20. Murrell DV, Lotze TE, Farber HJ, Crawford CA and Wiemann CM: The experience of families with children with spinal muscular atrophy type I across health care systems. J Child Neurol 32: 917-923, 2017.

21. Markowitz JA, Singh P and Darras BT: Spinal muscular atrophy: A clinical and research update. Pediatr Neurol 46: 1-12, 2012.

This work is licensed under a Creative Commons Attribution-NonCommercial-NoDerivatives 4.0 International (CC BY-NC-ND 4.0) License. 